\title{
The broad X-ray emission of the millisecond pulsar PSR J0437-4715
}

\author{
Sebastien Guillot ${ }^{1}$, et al. ${ }^{\dagger}$ \\ ${ }^{1}$ IA-PUC, Santiago, Chile \\ email: sguillot@astro.puc.cl; \\ ${ }^{\dagger}$ for the full list of co-authors and their affiliations, see Guillot et al. (2016).
}

\begin{abstract}
The nearest millisecond pulsar PSR J0437-4715 is the ideal target to constrain the dense matter equation of state using the lightcurve modelling method. Our analysis combining $X M M$-Newton, NuSTAR, and ROSAT observations removed ambiguities in the spectral modelling of the surface emission from PSR J0437-4715. Furthermore, the NuSTAR observation demonstrated that the non-thermal hard tail emission was pulsed at the pulsar spin period. These features are crucial to model the lightcurve and to measure the radius of the neutron star. This conference proceeding is based on the publication Guillot et al. (2016).
\end{abstract}

Keywords. stars: neutron, (stars:) pulsars: individual (PSR J0437-4715)

\section{Introduction}

PSR J0437-4715, the nearest millisecond pulsar (MSP), is a key target for investigation with the Neutron Star Interior Composition ExploreR (NICER, Gendreau et al., 2012). The accurate modeling of this MSP's lightcurve (and other MSPs) obtained with NICER, will result in measurements of the neutron star radius and constraints on the dense matter equation of state (Bogdanov et al., this volume). Furthermore, the precise timing of this MSP by the Parkes Radio Telescope permits knowing precisely its physical (mass, spin period, etc) and orbital properties (see Reardon et al. 2016, for all timing properties).

PSR J0437-4715 had been observed and detected at most wavelengths (Durant et al. 2013), from the radio bands, where one sees the pulsar's coherent emission, to the $\gamma$-ray energy range (non-thermal emission). In between, the white dwarf companion emits in the infrared and optical bands, and the neutron star polar cap emission is observed in the soft X-ray band. Finally, poorly constrained non-thermal, likely magnetospheric, emission is also visible in the Opt./UV regime and well as above $\sim 3 \mathrm{keV}$ in the $\mathrm{X}$-ray regime.

This work, published in Guillot et al. (2016), focused on understanding the broad X-ray emission of this pulsar, as well as the temporal properties of its non-thermal emission.

\section{Spectral analysis}

Our NuSTAR observation (215 ksec) of PSR J0437-4715 in the 3-20 keV hard X-ray band constrained the slope of the non-thermal emission (power-law index $\Gamma=1.5 \pm 0.25$ ). The handle on the power-law slope provided by $N u S T A R$, together with spectra from $X M M-N e w t o n$ and ROSAT, helped confirm the presence of a "cold" thermal component $\left(k T_{\mathrm{BB}} \sim 30 \mathrm{eV}\right)$ by removing ambiguities in the broad band X-ray emission (Fig. 1). This cold component presumably originates from the entire neutron star surface (except the hot polar caps). We note that the non-thermal component has a non-negligible contribution in the NICER band $(0.2-10 \mathrm{kev})$. 

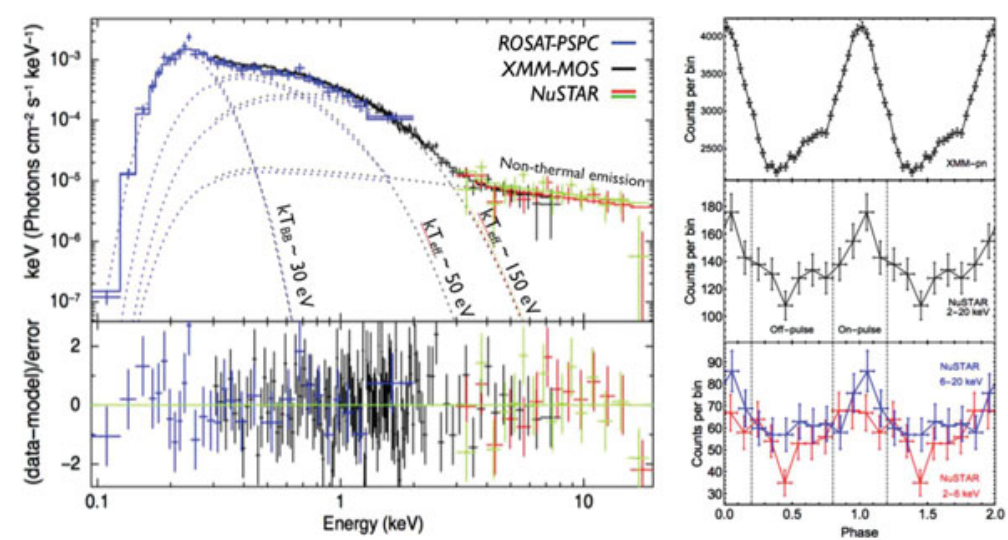

Figure 1. (left) Unfolded spectrum of PSR J0437-4715. (right) Pulse profile of PSR J0437-4715 with XMM-Newton (from Bogdanov 2013) and NuSTAR.

\section{Timing with NuSTAR}

Using the precise PSR J0437-4715 ephemeris from the Molonglo Telescope, we detect pulsations at the $3.7 \sigma$ level in the $2-20 \mathrm{keV}$ NuSTAR data. The observed pulsations in that energy range could be dominated by the polar cap emission $\left(k T_{\text {eff }} \sim 150 \mathrm{eV}\right)$. However, since such emission is negligible above $6 \mathrm{keV}$, we search for pulsations in the 6-20 keV range. We find some evidence of a pulsed signal from the non-thermal emission above $6 \mathrm{keV}$ at the $2.5 \sigma$ level. The low detection significance is possibly due to the known NuSTAR clock drift which smears out pulsations. Attempts to correct for this clock drift were unsuccessful with this data set, likely due to the pulsar's faintness and the pulse profile shape (see Gotthelf \& Bogdanov 2017, for a successful clock drift correction on pulsars with narrow pulse profiles).

\section{Conclusion}

We determined without ambiguities the presence of a cold thermal component, mostly present below $0.3 \mathrm{keV}$, from the surface of PSR J0437-4715. Knowing the surface temperature of old pulsars is important to understand re-heating mechanisms that maintain them hot (Gonzalez \& Reisenegger 2010). We also showed that the pulsar emission in the $N u S T A R$ band is likely pulsed at the neutron star spin period $(3.7 \sigma)$, and we tentatively detected X-ray pulsations above $6 \mathrm{keV}(2.5 \sigma)$ indicating that the non-thermal emission is also pulsed. This information is crucial for the radius measurements extracted from NICER data via lightcurve modelling (Miller \& Lamb 2016, Özel et al. 2016).

\section{Acknowledgement}

SG is a FONDECYT post-doctoral fellow, funded by grant \#3150428

\section{References}

Bogdanov, S. 2013, ApJ, 762, 96

Durant, M., Kargaltsev, O., \& Pavlov, G. G., et al. 2013, ApJ, 746, 6

Gendreau K. C., Arzoumanian Z., Okajima T. 2012, SPIE Conf. Series, 8443

Gonzalez, D. \& Reisenegger, A. 2010, A\& A, 522, A16

Gotthelf, E. V.\& Bogdanov, S. 2017, ApJ, 845, 159G

Guillot, S., Kaspi, V. M., \& Archibald, R. F., et al. 2016, MNRAS, 463, 2612-2622

Miller, M. C. \& Lamb, F. K. 2016, EPJA, 52, 63

Özel, F., Psaltis, D., \& Arzoumanian, Z. et al. 2016, ApJ, 832, 92

Reardon, D. J., et al. 2016, MNRAS, 455, 1751 